\title{
Stress effect on the kinetics of silicon thermal oxidation
}

\author{
Jui-Yuan Yen and Jenn-Gwo Hwu \\ Department of Electrical Engineering, Room 446, National Taiwan University, Taipei, Taiwan, \\ Republic of China
}

(Received 28 August 2000, accepted for publication 20 November 2000)

\begin{abstract}
Oxidation of silicon wafers under external mechanical stress was studied in this work. From the oxide thickness profile measured by an automatic-scanning ellipsometer, it was found that the oxidation kinetics of silicon were significantly affected by mechanical stress. There are two distinct features of oxide thickness distribution corresponding to short and long times. By comparing the kinetic constants taken from experiments and the simulated stress distribution on the silicon wafer, we can possibly explain the two features of oxide thickness distribution: the initial rate constant is deformation dependent and the parabolic rate constant is stress dependent. The observed stress-dependent oxidation rates are important in the study of thin gate oxide reliability. (C) 2001 American Institute of Physics. [DOI: 10.1063/1.1342801]
\end{abstract}

\section{INTRODUCTION}

With ultralarge-scale integration (ULSI) technology trends toward the deep-submicron regime, the reliability issue of mechanical stress becomes critical since the devices are much more complex in geometry and in the thin film materials used. ${ }^{1}$ Intrinsic stress generated during thermal oxidation is caused by the mismatch of molecular volume and thermal expansion coefficients between $\mathrm{Si}$ and $\mathrm{SiO}_{2} \cdot{ }^{2-5}$ This kind of stress is compressive in the oxide and tensile in silicon. ${ }^{6}$ Mechanical stress generally comes from the deposition of different kinds of films such as $\mathrm{SiO}_{2}$, poly-Si, $\mathrm{Si}_{3} \mathrm{~N}_{4}$ and metal electrodes. Structures of devices at sharp corners and at interconnect edges exhibit high stress as well. Mechanical stress causes not only dislocation of the silicon substrate and cracking or failure of thin films, but also the degradation of electrical characteristics. Problems of failure in integrated circuits can be ultimately traced to mechanical stress that occurs at various process steps. Therefore it is important to understand the nature and impact of stress.

There are many studies that have concentrated on the nonplanar silicon oxidation and stress-dependent oxidation kinetic theory by extending the well-known Deal-Grove model. ${ }^{7-11}$ It was also proposed that the compressive and tensile stresses from temperature nonuniformity or mechanically applied forces could affect the oxidation rate. ${ }^{12-14}$ However, there is still little discussion of the oxidation kinetics under stress in the existing literature. In this work, we studied the effect of external mechanical stress on silicon oxidation, especially for finding the relation between oxidation kinetic constants and applied stress based on experimental and simulation results. Our work demonstrates the substantial effect of stress since the linear and parabolic rate constants are deformation and stress dependent, respectively. The observation is partially consistent with the hypothesis of

\footnotetext{
a) Author to whom correspondence should be addressed; electronic mail: hwu@ee.ntu.edu.tw
}

the current stress-dependent model for silicon thermal oxidation.

\section{THEORETIC MODELS FOR SILICON THERMAL OXIDATION}

The silicon thermal oxidation mechanism had been explained by the well-known Deal-Grove model. ${ }^{15}$ This model showed that oxidation behavior could be represented by the expression $x^{2}+A x=B(t+\tau)$, where $x$ is the oxide thickness, $t$ is the oxidation time, and $\tau$ is the equivalent oxidation time of initial oxide thickness before oxidation. It exhibits two processes in this model. One process is the chemical reaction at the $\mathrm{Si}-\mathrm{SiO}_{2}$ interface and is represented by the linear rate constant $B / A$. Another process is the diffusion process of oxygen atom through the $\mathrm{SiO}_{2}$ film already formed and is represented by the parabolic rate constant $B$. The applicability of this model is restricted to onedimensional planar oxidation and is not completely suitable for the oxidation of a nonplanar or shaped silicon surface, ${ }^{7-11,16}$ even in the microscopic observation of onedimensional planar oxidation. ${ }^{17-20}$

Because the volume of a molecule of $\mathrm{SiO}_{2}$ is about 2.3 times that of a silicon atom, ${ }^{11,13,21}$ silicon oxidation is accompanied by a large increase in volume, thus large mechanical stress would be generated during oxidation. If the volume expansion only takes place normal to the silicon surface, no stress will be generated along the planar direction. However, experimental evidence has shown that the silicon wafers become curved during oxidation. ${ }^{2-5}$ This indicates the existence of fairly large in-plane stress in the $\mathrm{SiO}_{2}$ film. The large in-plane stress is believed to have a substantial effect on the oxidation behavior, either on diffusion of the oxygen atom through the $\mathrm{SiO}_{2}$ film or on the chemical reaction at the $\mathrm{Si}-\mathrm{SiO}_{2}$ interface. ${ }^{11,18,22}$

Some complicated numerical models for nonuniform silicon oxidation have been developed to explain the microscopic oxidation behavior by extending the well-known Deal-Grove model to a two-dimensional stress-dependent model. ${ }^{7-11}$ These modern oxidation models propose three 


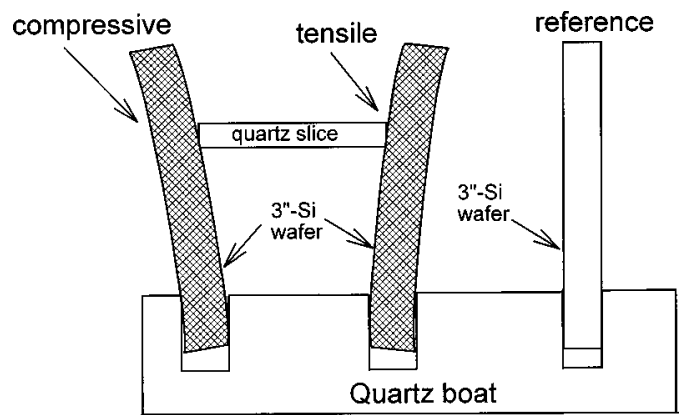

FIG. 1. Experimental setup of furnace oxidation with tensile and compressive stress applied on wafers.

critical oxidation parameters that are stress dependent. The three parameters are surface reaction rate $k_{s}$, oxidant diffisivity $D$, and oxide viscosity $\eta$.

\section{EXPERIMENT}

Boron-doped (100), 3 in. silicon wafers with a resistivity of $1-10 \Omega \mathrm{cm}$ were used in this experiment. After standard RCA cleaning, the wafers were furnace oxidized in pure dry $\mathrm{O}_{2}$ at $950{ }^{\circ} \mathrm{C}, 1 \mathrm{~atm}$. Conventional furnace thermal oxidation was used here in order to prevent temperature nonuniformity. Figure 1 shows a schematic diagram of the experimental setup. The wafers were placed vertically on a quartz boat. A small slice of quartz about $20 \mathrm{~g}$ was put across the middle of two adjacent wafers so that the two adjacent wafers sustain compressive and tensile stress separately. External applied force was applied so as not to cause visible dislocations or defects on the silicon wafers. This can be examined by using a preferential silicon etching solution to the etch silicon wafer after oxide removal. ${ }^{23-26}$ After oxidation, the oxide thickness was measured by an automatic-scanning ellipsometer with an accuracy of $\pm 5 \AA$.

Figures 2 and 3 show the oxide thickness profiles of the silicon wafer under compressive and tensile stress at different oxidation times, respectively. The data were measured at a $3 \mathrm{~mm}$ pitch so that one could see the entire oxide thickness profile on each silicon wafer. From Fig. 3(a), one can see that the wafer with tensile stress has a visible increase in oxide thickness. This phenomenon was mainly caused by the enlarged atom spacing of the silicon wafer under tensile stress at the initial oxidation region, as was discussed in previous studies. ${ }^{13,14}$ From Figs. 3(b) and 3(c), we found that when the oxidation time was longer, the oxide thickness at the lower part of the silicon wafer was somewhat thicker than that in Fig. 3(a). In order to realize this change in feature of the oxide thickness distribution, we extracted the kinetic rate constants from data fitting according to the well-known Deal-Grove model and compared them with the simulation stress distribution, discussed later. In this work, the experimental results of tensile stress showed an obvious effect on oxide thickness while that of compressive stress in Fig. 2 did not have a significant effect. The latter was mainly due to the fact that compressive stress was applied on the back side of silicon wafer.
Compressive Stress

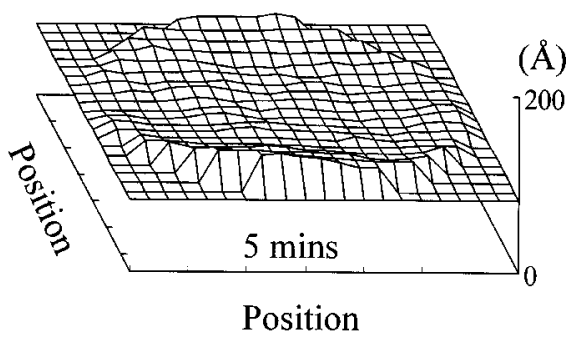

(a)
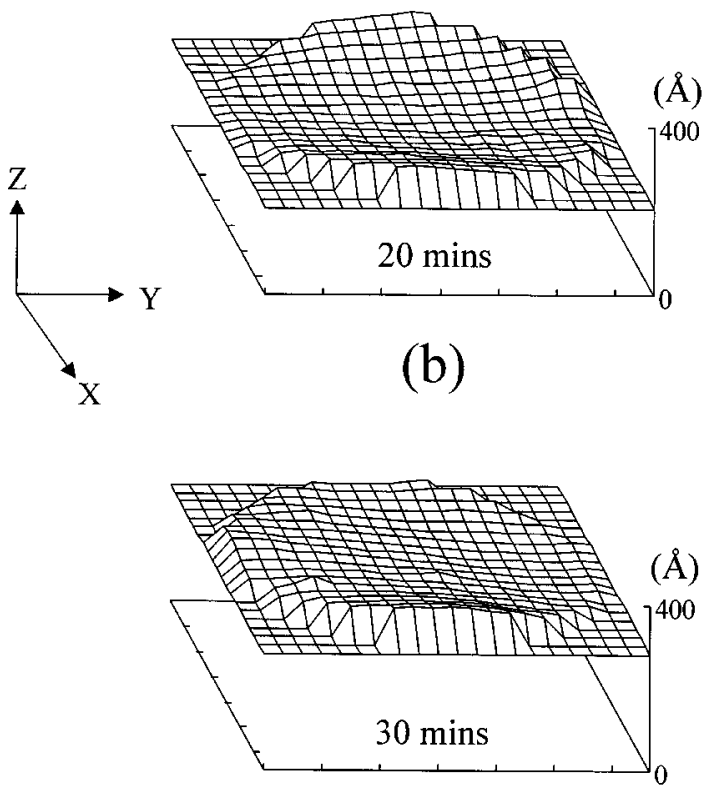

(c)

FIG. 2. Oxide thickness distributions under compressive stress for oxidation times of (a) 5, (b) 20, and (c) $30 \mathrm{~min}$.

\section{STRESS SIMULATION}

ANSYS $^{\oplus}$ Ver. 5.5 simulation software based on the finiteelement method was used in this simulation. The simulation simply considered the silicon wafer under stress during oxidation. Planar stress conditions with stress $\sigma_{z}=0$ but deformation $U_{z} \neq 0$ and isotropic material properties were assumed. The stress was applied in the $-Z$ direction across the middle of the silicon wafer in the $X$ direction. The bottom part of the silicon wafer was assigned to no displacement because it was constrained by the quartz wafer boat. That is, the wafer was absolutely rigid at the bottom of the boat in our simulation, although we did not know the actual size of the Si wafer constrained by the quartz boat. There was no other boundary condition or assumption in this simplified modeling. Figure 4 shows a diagram of the simulation setup. The material properties of the silicon wafer and the experimental parameters were as follows: Young's modulus $E_{\mathrm{Si}}=130 \mathrm{GPa}$, Poisson ratio $\nu_{\mathrm{Si}}=0.28$, shear modulus $G$ $=80$, density $\sigma=2330 \mathrm{~kg} / \mathrm{m}^{3}$, linear thermal expansion coefficient $\alpha=4.2 \times 10^{-6} /{ }^{\circ} \mathrm{C}\left(25^{\circ} \mathrm{C}\right)$, reference temperature $T_{0}$ $=25^{\circ} \mathrm{C}$, and temperature load $\Delta T=925^{\circ} \mathrm{C} .{ }^{11,27,28}$ 


\section{Tensile Stress}

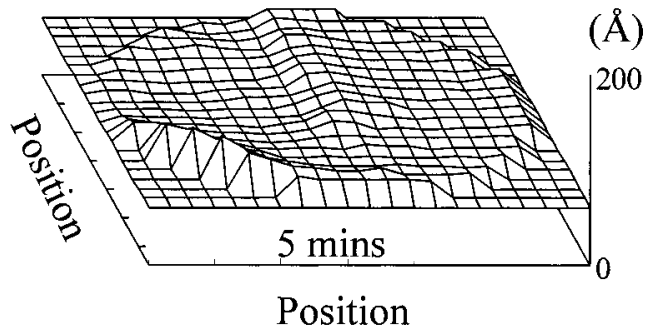

(a)
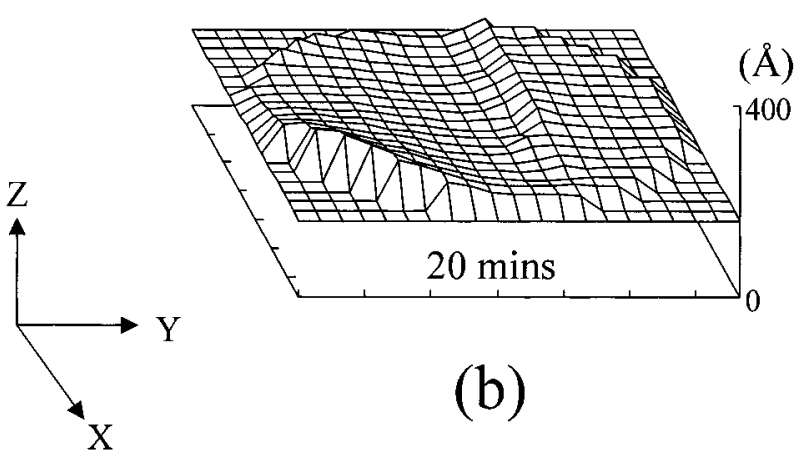

(b)

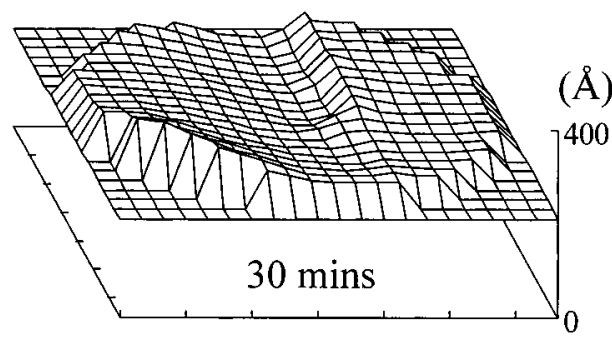

(c)

FIG. 3. Oxide thickness distributions under tensile stress for oxidation times of (a) 5, (b) 20, and (c) $30 \mathrm{~min}$.

The temperature load was the temperature difference between the reference temperature, $25^{\circ} \mathrm{C}$, and the oxidation temperature, $950^{\circ} \mathrm{C}$. The force applied in the $-Z$ direction generates deformation and mechanical stress in the $X$ and $Y$ directions. According to the simulation carried out under the above applied force and temperature loads, we could obtain deformation components $U_{x}, U_{y}, U_{z}$, and stress components $\sigma_{x}, \sigma_{y}, \tau_{x y}$ of silicon. We put these stress components into the current stress-dependent oxidation model to compare with our experimental results. Figures 5(a) and 5(b) show the simulated deformation $U_{x y}=U_{x} U_{y}$, i.e., the product of deformation in the $X$ and $Y$ directions, and the pressure $p_{\mathrm{Si}}$ $=1 / 3\left(\sigma_{x(\mathrm{Si})}+\sigma_{y(\mathrm{Si})}+\sigma_{z(\mathrm{Si})}\right)$ in silicon, respectively. Clearly, nonuniform distributions of deformation and stress on the wafer due to intentionally induced mechanical stress are observed.

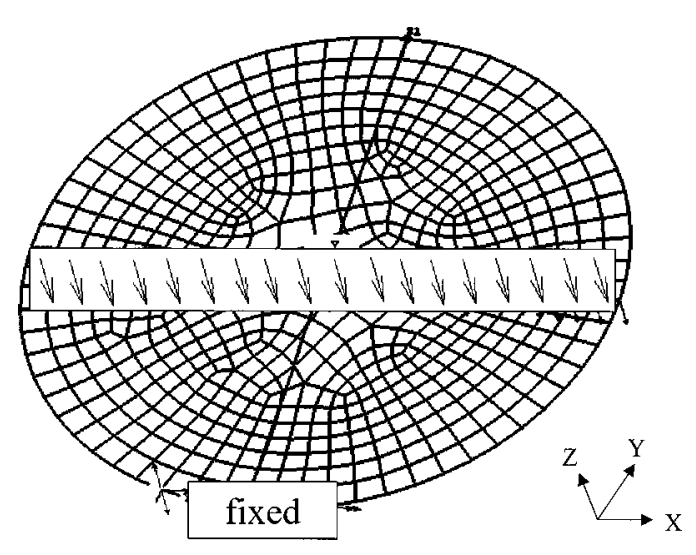

FIG. 4. Diagram of stress simulation setup by using ANSYS ${ }^{\odot}$ Ver. 5.5 simulation software.

\section{RESULTS AND DISCUSSION}

\section{A. Linear rate constant}

Figure 6(a) shows three measurement points of oxide, $s 11, s 14$, and $s 17$, on the silicon wafer under tensile stress, and Fig. 6(b) shows their measured oxide thickness versus oxidation time as well as the fitting polynomial curves for these three points. We could extract the linear and parabolic rate constants from the polynomial fitting curves in Fig. 6(b) according to the well-known Deal-Grove model. Figure 7(a)

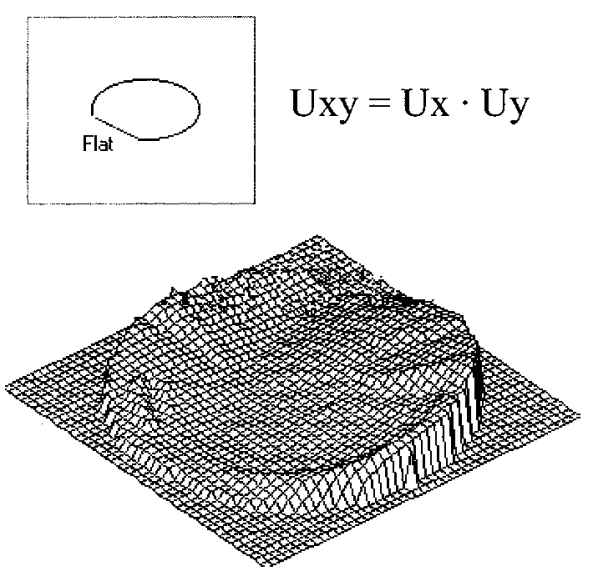

(a)

$$
p_{\mathrm{Si}}=1 / 3\left(\sigma_{\mathrm{x}(\mathrm{Si})}+\sigma_{\mathrm{y}(\mathrm{Si})}+\sigma_{\mathrm{z}(\mathrm{Si})}\right)
$$

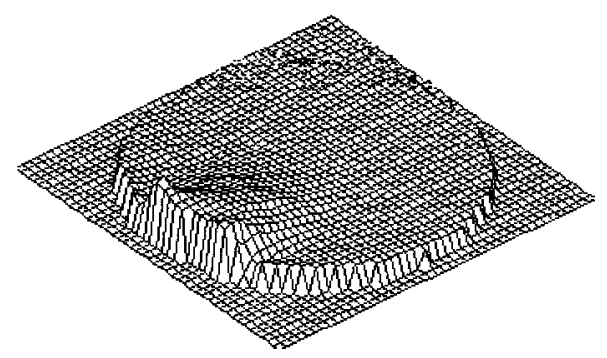

(b)

FIG. 5. Simulated (a) deformation distribution and (b) stress distribution on a silicon wafer under tensile stress. 


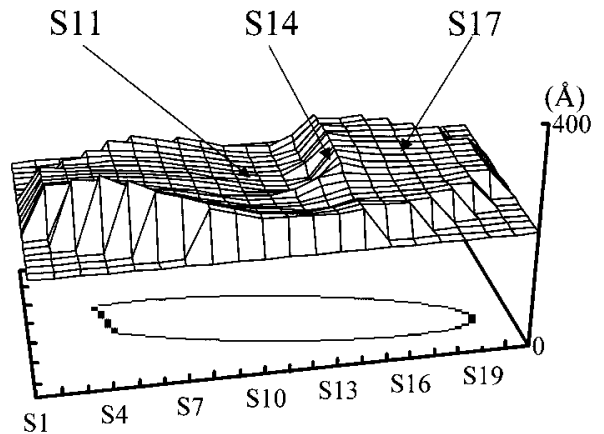

(a)

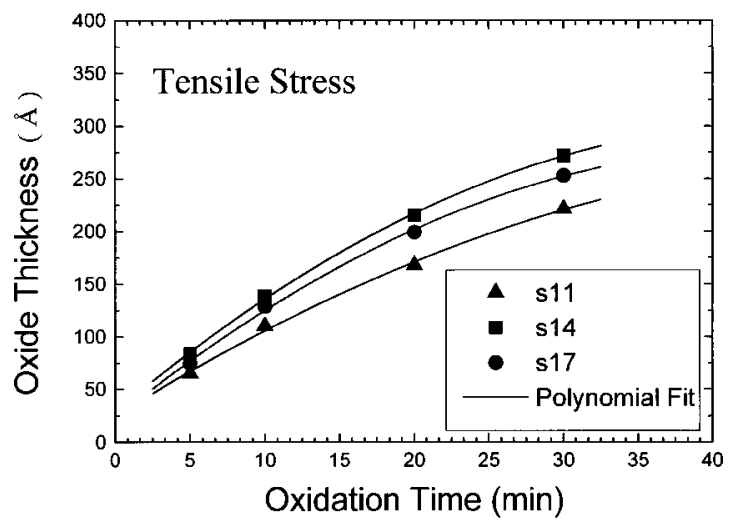

(b)

FIG. 6. (a) Three measurement points of oxide on the silicon wafer under tensile stress and (b) their measured oxide thickness vs oxidation time as well as the fitting polynomial curves for these three points.

shows the linear rate constant $B / A$ versus the position across the diameter of silicon wafers under tensile stress and without stress, respectively. The linear rate constant $B / A$ represents the chemical reaction at the $\mathrm{Si}-\mathrm{SiO}_{2}$ interface and has an enhanced value in the upper portion of the silicon wafer. From the experimental result shown in Figs. 3(a)-3(c), the oxide thickness distributions of the silicon wafer under tensile stress also exhibit a similar enhancement feature. It is proposed that this phenomenon is related to deformation of the silicon wafer since the spacing of silicon atoms is a main factor in determining the oxidation rate in the initial oxidation regime. From Figs. 3(a)-3(c), the portion of the silicon wafer to which stress was applied has the largest deformation in the wafer, therefore there is increasing oxide thickness in the middle of silicon wafer.

Before the temperature load, the simulated deformation in the $y$ direction $U_{y}$ is much larger than that in the $X$ direction $U_{x}$. The former possibly dominates the oxide growth rate in the initial oxidation regime and the observed phenomenon has been discussed previously. ${ }^{12}$ However, after the temperature load $\Delta T=925^{\circ} \mathrm{C}$ was applied in the simulation, there is a significant increase of $U_{x}$ and $U_{y}$ due to thermal expansion of the silicon wafer. Figure 7(b) shows the simulated $U_{x y}$, i.e., the product of $U_{x}$ and $U_{y}$, versus the position across the center diameter of silicon wafer. The product of $U_{x}$ and $U_{y}$ represents the expanding unit area of the silicon wafer under the temperature load. From Figs. 7(a) and 7(b),

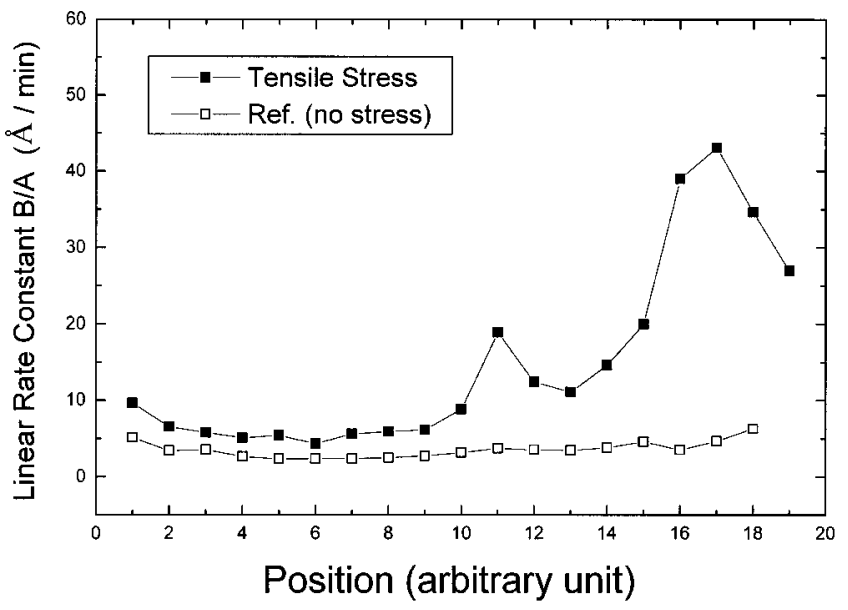

(a)

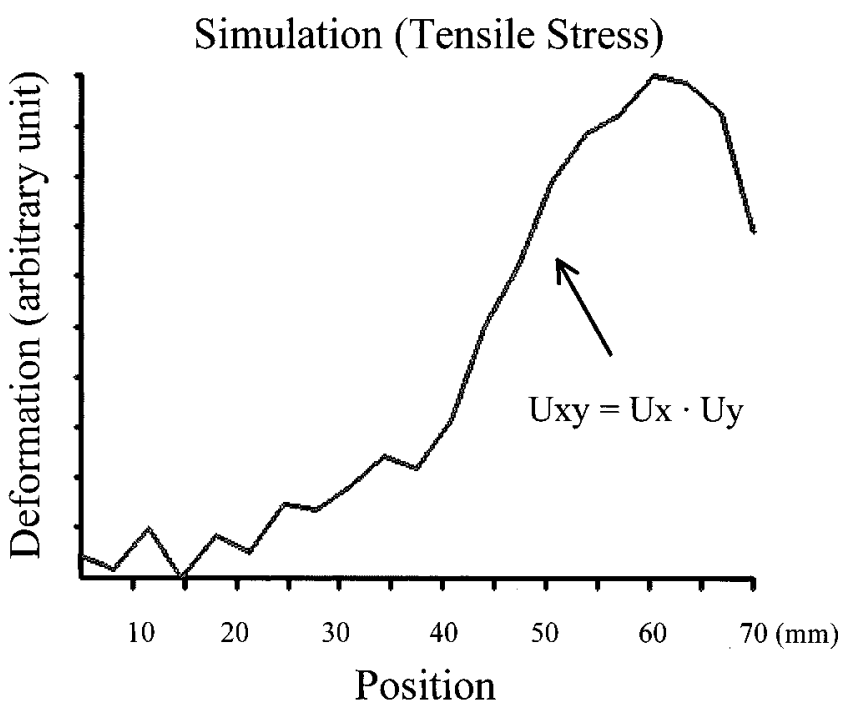

(b)

FIG. 7. (a) Experimental linear rate constant $B / A$ across the wafer and (b) simulated deformation distribution on a wafer under tensile stress.

it is believed that the expanding unit area of the silicon wafer under tensile stress and temperature load is related to the experimental linear rate constant $B / A$.

\section{B. Parabolic rate constant}

Figure 8(a) shows the parabolic rate constant $B$, extracted from experimental data fitting according to the wellknown Deal-Grove model, versus the position across the center diameter of silicon wafer under tensile stress. The parabolic rate constant $B$ represents the diffusion process of the oxygen atom through the $\mathrm{SiO}_{2}$ film and has an enhanced feature in the lower portion of the silicon wafer. The enhancement feature of oxide thickness can be seen in Figs. $3(a)-3(c)$ when the oxidation time is long. When the oxidation time is short, only in the middle portion of the silicon wafer is there a visible increase in oxide thickness.

According to the current stress-dependent model of silicon oxidation, it has been hypothesized that one of the ef- 


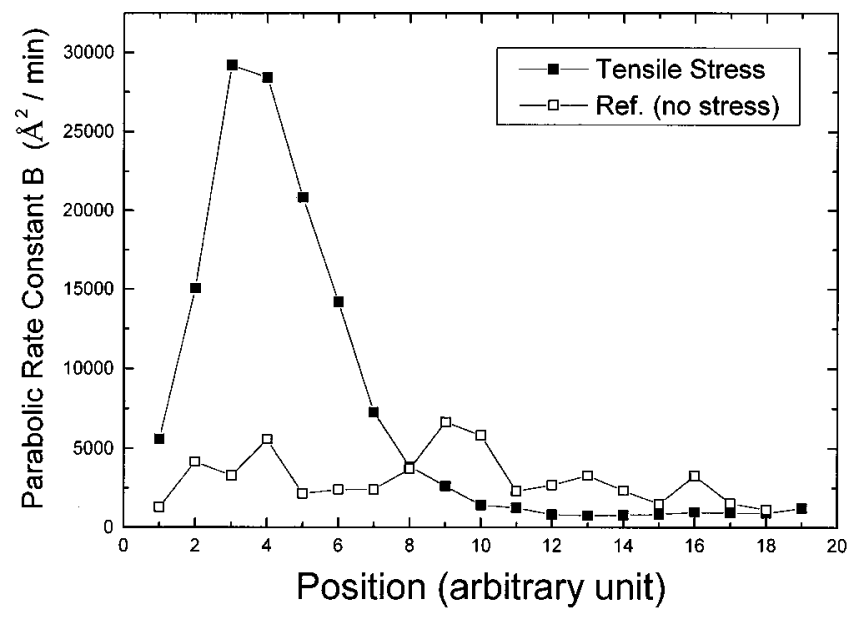

(a)

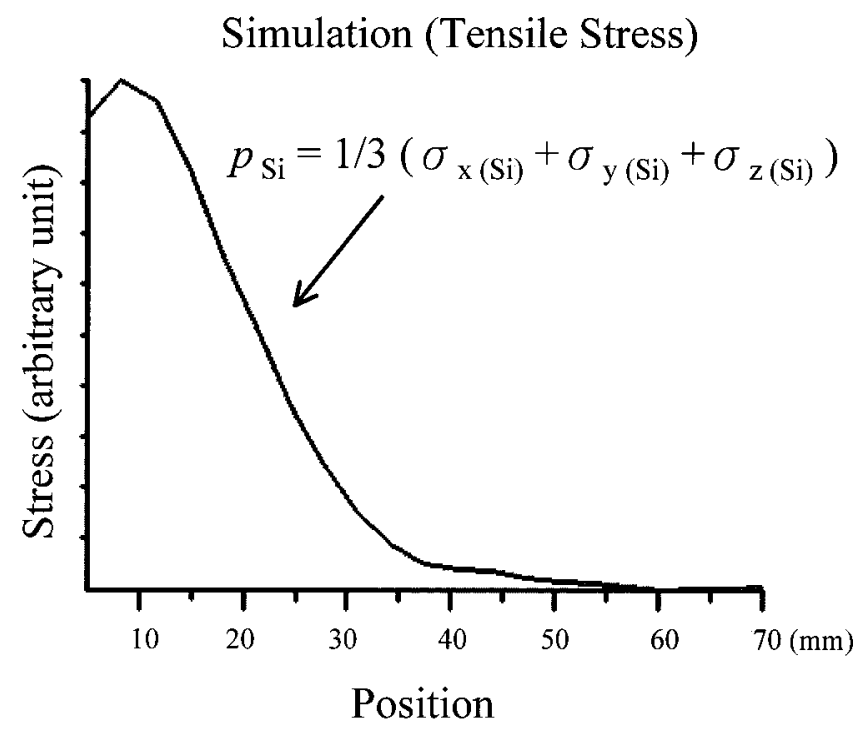

(b)

FIG. 8. (a) Experimental parabolic rate constant $B$ across the wafer and (b) simulated deformation distribution on a wafer under tensile stress.

fects of stress on $\mathrm{SiO}_{2}$ film is the change of diffusivity in the oxidant. The parameter of oxidant diffusivity $D$ versus the hydrostatic pressure $p$ is $^{7-11}$

$$
D=D_{0} \exp \left(-p_{\mathrm{SiO}_{2}} V_{d} / k T\right),
$$

where $p$ is the hydrostatic pressure in the $\mathrm{SiO}_{2}$ and is equal to the average of normal stress components in the $\mathrm{SiO}_{2}$, i.e., $p_{\mathrm{SiO}_{2}}=1 / 3\left(\sigma_{x\left(\mathrm{SiO}_{2}\right)}+\sigma_{y\left(\mathrm{SiO}_{2}\right)}+\sigma_{z\left(\mathrm{SiO}_{2}\right)}\right)$, where $\sigma_{x\left(\mathrm{SiO}_{2}\right)}$, $\sigma_{y\left(\mathrm{SiO}_{2}\right)}$, and $\sigma_{z\left(\mathrm{SiO}_{2}\right)}$ are stress components in the $X, Y$, and $Z$ directions of $\mathrm{SiO}_{2}$, respectively. $D_{0}$ is the zero-stress diffusion coefficient. $V_{d}$ is a fitting parameter of the selected volume. Equation (1) predicts increasing $D$ when $p$ is negative. The increasing oxidant diffusivity $D$ will make the oxidation rate faster.

The stress-dependent model also proposes the oxidation behavior on a nonuniform silicon surface. When oxide is grown on a convex silicon surface, the old oxide is stretched as it is being pushed out. In such a case the $\mathrm{SiO}_{2}$ film is in a state of tension, so the pressure $p$ in the $\mathrm{SiO}_{2}$ is tensile and negative in Eq. (1). When oxide is grown on a concave silicon surface, the oxide is compressed as it is pushed in. The $\mathrm{SiO}_{2}$ film is in a state of compression, so the pressure $p$ in the oxide is compressive and positive in Eq. $(1) \cdot{ }^{8-10}$

At present, there is no suitable stress-dependent model for silicon oxidation with applied stress as in our experiment. According to another existing theory and to experimental evidence, ${ }^{2-6}$ the silicon surface generally bends convexly after thermal oxidation, i.e., the silicon is in a state of tensile stress and the $\mathrm{SiO}_{2}$ is in a state of compression. However, this theory is derived taking into consideration intrinsic stress and thermal stress which were generated from mismatch of the molecular volume and the different thermal expansion coefficients between $\mathrm{Si}$ and $\mathrm{SiO}_{2}$ during thermal oxidation. In this work, we consider only the stress caused by the applied force and the temperature load on silicon, without calculating the intrinsic stress and thermal stress. We also consider that our experimental sample under tensile stress was in a convex surface after oxidation because the sample was given a positive feedback of convex due to applied tensile stress. Therefore it is reasonable to assume that our experiment is likely to be the case of oxidation which can be explained by the above stress-dependent model.

According to the above discussions, we have tried to use Eq. (1) to explain our experimental result and simulation. Our simulation showed that after oxidation under tensile stress on the silicon wafer, the stress components $\sigma_{x(\mathrm{Si})}$ and $\sigma_{y(\mathrm{Si})}$ in silicon were both tensile. We cited the concept from the stress-dependent model that in the convex silicon surface, the pressure $p$ in the $\mathrm{SiO}_{2}$ was tensile and negative in Eq. (1). Equation (1) predicts increasing oxidant diffisivity $D$ when $p$ is negative. We defined a modified pressure $p_{\mathrm{Si}}=1 / 3\left(\sigma_{x(\mathrm{Si})}\right.$ $\left.+\sigma_{y(\mathrm{Si})}+\sigma_{z(\mathrm{Si})}\right)$ in the silicon and use $p_{\mathrm{Si}}$ to represent the pressure $p_{\mathrm{SiO}_{2}}=1 / 3\left(\sigma_{x\left(\mathrm{SiO}_{2}\right)}+\sigma_{y\left(\mathrm{SiO}_{2}\right)}+\sigma_{z\left(\mathrm{SiO}_{2}\right)}\right)$ in the $\mathrm{SiO}_{2}$. This is on the basis that we could derive the stress in the oxide from the stress in the silicon according to the equilibrium theory of fluid. ${ }^{2,6,29-31}$ In our two-dimensional simulation, only $\sigma_{x(\mathrm{Si})}$ and $\sigma_{y(\mathrm{Si})}$ are of interest and $\sigma_{z(\mathrm{Si})}=0$, thus only $\sigma_{x\left(\mathrm{SiO}_{2}\right)}$ and $\sigma_{y\left(\mathrm{SiO}_{2}\right)}$ are effective and $\sigma_{z\left(\mathrm{SiO}_{2}\right)}=0$.

Figure $8(\mathrm{~b})$ shows the simulated pressure of $\mathrm{Si}$ under tensile stress $p_{\mathrm{Si}}$ versus positions on the silicon wafer. From Fig. 8(b), we could infer that, when $\mathrm{Si}$ is under tensile stress, the $p_{\mathrm{SiO}_{2}}$ in Eq. (1) is negative and therefore the oxidant diffisivity $D$ becomes large. The large oxidant diffisivity $D$ would enhance the oxidant diffusion process, so the parabolic rate constant $B$ became large as shown in Fig. 8(a). It is believed that the simulation is consistent with the observed behavior of experimental parabolic rate constant $B$, shown in Fig. 8(a). Our results are also consistent with previously published work that suggests that the main effect of stress is on the parabolic rate constant. ${ }^{22}$

\section{CONCLUSION}

Silicon oxidation with externally applied mechanical stress is useful in studying the mechanical stress-induced oxidation rate. By comparing the kinetic constants extracted 
from experiments and the simulated stress distribution on the silicon wafer, we find important features like the linear rate constant $B / A$ is deformation dependent and the parabolic rate constant $B$ is stress dependent. It is therefore of interest to note that the oxidation kinetic rate constants are not only temperature dependent but are also affected by stress. This is important in the investigation of oxide quality related to stress especially in the ultrathin oxide regime.

\section{ACKNOWLEDGMENT}

This work was supported by the National Science Council of the Republic of China under Contract No. NSC 892215-E-002-042.

${ }^{1}$ S. M. Hu, J. Appl. Phys. 70, R53 (1991).

${ }^{2}$ R. J. Jaccodine and W. A. Schlegel, J. Appl. Phys. 37, 2429 (1966).

${ }^{3}$ E. P. EerNisse, Appl. Phys. Lett. 30, 290 (1977).

${ }^{4}$ E. P. EerNisse, Appl. Phys. Lett. 35, 8 (1979).

${ }^{5}$ T. Y. Tan and U. Gösele, Appl. Phys. Lett. 39, 86 (1981).

${ }^{6}$ E. P. Jacobs and G. Dorda, Surf. Sci. 73, 357 (1978).

${ }^{7}$ H. Matsumoto and M. Fukuma, IEEE Trans. Electron Devices ED-32, 132 (1985).

${ }^{8}$ D. B. Kao, J. P. McVittie, W. D. Nix, and K. C. Saraswat, IEEE Trans. Electron Devices ED-34, 1008 (1987).

${ }^{9}$ D. B. Kao, J. P. McVittie, W. D. Nix, and K. C. Saraswat, IEEE Trans. Electron Devices ED-35, 25 (1988).
${ }^{10}$ P. Sutardia and W. G. Oldham, IEEE Trans. Electron Devices ED-36, 2415 (1989)

${ }^{11}$ T. J. Delph, J. Appl. Phys. 83, 786 (1998).

${ }^{12}$ A. Minhalyi, R. J. Jaccodine, and T. J. Delph, Appl. Phys. Lett. 74, 1981 (1999).

${ }^{13}$ R. Deaton and H. Z. Massoud, J. Appl. Phys. 70, 3588 (1991).

${ }^{14}$ J.-Y. Yen and J.-G. Hwu, Appl. Phys. Lett. 76, 1834 (2000).

${ }^{15}$ B. E. Deal and A. S. Grove, J. Appl. Phys. 36, 3770 (1965).

${ }^{16}$ R. B. Marcus and T. T. Sheng, J. Electrochem. Soc. 129, 1278 (1982).

${ }^{17}$ E. A. Irene, D. W. Dong, and R. J. Zeto, J. Electrochem. Soc. 127, 396 (1980).

${ }^{18}$ C. H. Hsueh and A. G. Evans, J. Appl. Phys. 54, 6672 (1983).

${ }^{19}$ A. Fargeix, G. Ghibaudo, and G. Kamarinos, J. Appl. Phys. 54, 2878 (1983).

${ }^{20}$ A. Fargeix and G. Ghibaudo, J. Appl. Phys. 54, 7153 (1983).

${ }^{21}$ E. A. Irene, E. Tierney, and J. Angiello, J. Electrochem. Soc. 129, 2594 (1982).

${ }^{22}$ A. Fargeix and G. Ghibaudo, J. Appl. Phys. 56, 589 (1984).

${ }^{23}$ F. S. d'Aragona, J. Electrochem. Soc. 119, 948 (1972).

${ }^{24}$ M. W. Jenkins, J. Electrochem. Soc. 124, 757 (1977).

${ }^{25}$ D. G. Schimmel, J. Electrochem. Soc. 126, 479 (1979).

${ }^{26}$ K. H. Yang, J. Electrochem. Soc. 131, 1140 (1984).

${ }^{27}$ S. M. Sze, VLSI Technology, 2nd ed. (McGraw-Hill, New York, 1988).

${ }^{28}$ Y.-L. Shen, J. Mater. Res. 12, 2219 (1997).

${ }^{29}$ M. V. Whelan, A. H. Goemans, and L. M. C. Goossen, Appl. Phys. Lett. 10, 262 (1967).

${ }^{30}$ G. Charitat and A. Martinez, J. Appl. Phys. 55, 909 (1984).

${ }^{31}$ L. M. Mack and A. Reisman, J. Electrochem. Soc. 136, 3433 (1989). 